\title{
. \\ RELEVANCIA DE LAS COMPENTENCIAS GENÉRICAS IDENTIFICADAS EN EL CONTEXTO LABORAL Y VIVENCIAL DE LOS ESTUDIANTES
}

\section{RELEVANCE OF GENERIC COMPETENCES IDENTIFIED IN THE LABOR AND LIVING CONTEXT OF STUDENTS}

\author{
Autor 1. Wilington Ferney Rojas González \\ Ingeniero Industrial, especialista en Gerencia de mercadeo estratégico y \\ Magister en Educación, Institución y grupo de investigación \\ ORCID: https://orcid.org/0000-0002-9296-4979 \\ Correo electrónico: willingtonagel@gmail.com - wilington.rojas@unad.edu.co
}

\begin{abstract}
Autor 2. José Alfonso Mendoza Gallego
Administrador Bancario y Financiero, Tecnólogo en Gestión Comercial y Negocios y Magister en Educación de la Diversidad, Docente Investigador Grupo YUMA. Facultad de Ciencias Empresariales, Corporación Universitaria Minuto de Dios.
\end{abstract}

ORCID: https://orcid.org/0000-0002-0002-4104

Correo electrónico: Josemendozagallego@hotmail.com jmendozaga2@uniminuto.edu.co

\section{RESUMEN}

La investigación desarrolló el tema de competencias genéricas de los estudiantes del programa de administración de empresas de la Universidad Minuto de Dios. El objetivo fue analizar las estrategias pedagógicas de los docentes y estudiantes del programa de Administración de Empresas de la Universidad Minuto de Dios, para describir el uso de las competencias genéricas, aplicar los conocimientos en la práctica, resolver problemas y tomar decisiones, de los conocimientos adquiridos por los estudiantes y su transferencia al contexto vivencial.

En la investigación se empleó el enfoque cualitativo acompañado del método etnográfico, porque se buscó analizar un fenómeno social, asociado al uso de las competencias genéricas y la transferencia de los conocimientos adquiridos a su contexto vivencial de los estudiantes. A la población objeto de estudio se les aplicó una guía de observación y una entrevista semiestructurada, que permitió recolectar la información, analizarla e interpretarla; para llegar a los hallazgos y resultados. Los hallazgos de esta investigación confirmaron lo imperioso de trabajar las competencias en el aula; además dejó en evidencia lo importante que es desarrollar en los estudiantes competencias genéricas tales como: aplicar los conocimientos en la práctica, resolver problemas y tomar decisiones.

A partir de lo observado en los estudiantes en el trabajo de campo, estas competencias son apropiadas, para su contexto laboral y vivencial. Por último, se concluyó que las estrategias pedagógicas realizadas por los docentes para desarrollar las competencias genéricas en el aula le facilitan al educando, ya sea de forma individual o en equipo, trabajar el pensamiento crítico a partir del análisis de un problema o de una tomada de decisión; así mismo, la aplican los conocimientos previos y adquiridos. 




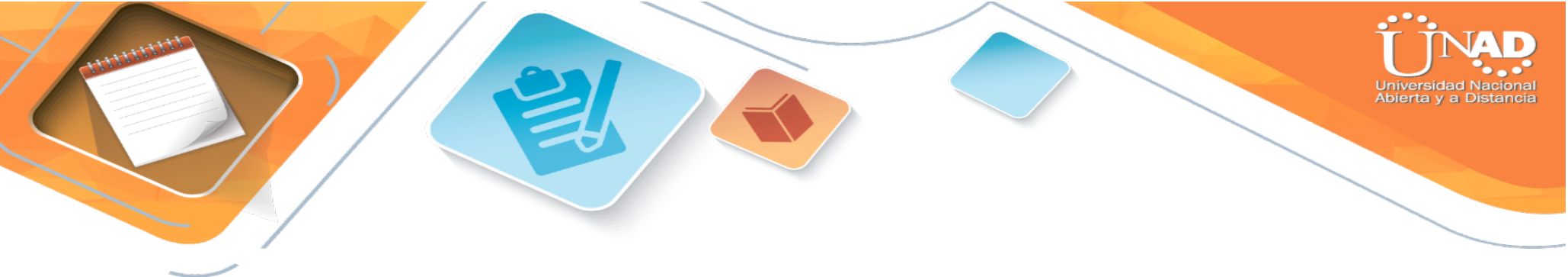

investigación" (Mayan, 2001). En este orden de ideas, el enfoque cualitativo, que explora desde las perspectivas de los participantes y desde sus experiencias, es el idóneo para alcanzar los objetivos de este estudio, porque facilita analizar, describir e identificar el uso de las competencias genéricas y la transferencia de los conocimientos adquiridos a su contexto vivencial de los estudiantes de Administración de Empresas de la Universidad Minuto de Dios de la ciudad de Neiva, Huila.

La técnica de recolección de información fue el método etnográfico porque intenta analizar un fenómeno social asociado al uso de las competencias genéricas y la transferencia de los conocimientos adquiridos a su contexto vivencial de los estudiantes. En este caso la etnografía es pertinente porque aborda el estudio desde "una concepción y práctica de conocimiento que busca comprender los fenómenos sociales desde la perspectiva de sus miembros, entendidos como "actores", "agentes" o "sujetos sociales." (Guber, 2001, p. 11).

De igual manera, La observación participante posibilita registrar los acontecimientos para su posterior análisis, puesto que "consiste en el registro sistemático, válido y confiable de comportamientos o conductas manifiestos y en la que el observador interactúa con los sujetos observados." (Fernández y Baptista, 2003, p. 428); y la entrevista semiestructurada, Entrevistar a cada uno de los participantes "permitió introducir preguntas adicionales para precisar conceptos u obtener más información sobre los temas deseados" (Sampieri, 2010)

Por lo anterior, se diseñaron los siguientes instrumentos: una guía de observación, y un guion para la entrevista que tenían como objetivo identificar y describir las competencias genéricas que desarrollan, los estudiantes de administración de empresas, para transferir sus conocimientos a su contexto vivencial. Finalmente, lo observado durante las prácticas fue registrado en matrices de análisis realizadas para la investigación con el fin de sistematizar la información necesaria para cumplir con el objetivo del estudio.

La selección de los participantes "sustenta la representatividad de un universo, se presenta como el factor crucial para generalizar los resultados" (Alvarez-Gayou, 2003). Para el presente proyecto, se seleccionó a estudiantes de administración de empresas, jornada nocturna, de la Corporación Universitaria Minuto de Dios, sede Neiva, Huila. Este curso seleccionado, estuvo conformado por 15 estudiantes y un docente; debido a que el número de estudiantes es pequeño se decidió realizar el proyecto con el $100 \%$ del curso.

\section{DISCUSIÓN Y RESULTADOS.}

En la sistematización y análisis de los resultados, se resaltan 4 categorías en relación con algunas competencias genéricas. Por lo anterior, se presentan los resultados:

\section{Análisis Descriptivo e Interpretación de Resultados}


A continuación, está la guía de observación con el número de estudiantes según la categoría en la que fueron calificados cada uno. La escala de la calificación de la guía según el desempeño está representada por las letras $A, B$ y $C$ y cada una significa lo siguiente: $A$ : INSATISFACTORIO. B: BUENO. C: EJEMPLAR.

Tabla 1

Resultados obtenidos de la observación de los estudiantes

\begin{tabular}{|c|c|c|c|c|c|c|}
\hline \multirow[t]{2}{*}{ No } & \multirow[t]{2}{*}{ Categorías } & \multirow[t]{2}{*}{ Subcategorías } & \multirow[t]{2}{*}{ Contenidos de Análisis } & \multicolumn{3}{|c|}{$\begin{array}{c}\text { Criterios } \\
\text { calificación }\end{array}$} \\
\hline & & & & $\mathbf{A}$ & B & C \\
\hline \multirow{2}{*}{1} & \multirow{2}{*}{$\begin{array}{l}\text { Resolución } \\
\text { de } \\
\text { problemas }\end{array}$} & \multirow{2}{*}{ Estrategias } & $\begin{array}{l}\text { El estudiante propone } \\
\text { solución a problemas según } \\
\text { quienes sean } \quad \text { sus } \\
\text { interlocutores y el contexto. }\end{array}$ & 1 & 4 & 10 \\
\hline & & & $\begin{array}{l}\text { El estudiante utiliza el proceso } \\
\text { apropiado para solucionar } \\
\text { situaciones } \\
\text { complejas. }\end{array}$ & 1 & 4 & 10 \\
\hline \multirow[t]{2}{*}{2} & \multirow{2}{*}{$\begin{array}{l}\text { Aplicación } \\
\text { de } \\
\text { conocimientos }\end{array}$} & \multirow[t]{2}{*}{ Transferencia } & $\begin{array}{llr}\text { El estudiante } & \text { aplica } & \text { los } \\
\text { conocimientos } & \text { previos } & \text { y } \\
\text { adquiridos } & \text { para } & \text { la } \\
\text { consecución de los objetivos } \\
\text { propuestos. }\end{array}$ & 1 & 4 & 10 \\
\hline & & & $\begin{array}{l}\text { El estudiante hace uso de los } \\
\text { conocimientos adquiridos, a la } \\
\text { hora de tomar de decisiones } \\
\text { complejas. }\end{array}$ & 1 & 4 & 10 \\
\hline \multirow{2}{*}{3} & \multirow{2}{*}{$\begin{array}{l}\text { Toma de } \\
\text { decisiones }\end{array}$} & \multirow{2}{*}{ Autonomía } & $\begin{array}{l}\text { El estudiante es capaz de } \\
\text { tomar decisiones sencillas y } \\
\text { coherentes y sabe justificarlas } \\
\text { con criterio. }\end{array}$ & 1 & 2 & 12 \\
\hline & & & $\begin{array}{l}\text { El estudiante toma la } \\
\text { iniciativa y es eficaz en la } \\
\text { toma de decisiones acertadas } \\
\text { en situaciones complejas. }\end{array}$ & 1 & 2 & 12 \\
\hline \multirow[t]{2}{*}{4} & \multirow[t]{2}{*}{ Aprendizaje } & \multirow[t]{2}{*}{ Significativo } & $\begin{array}{l}\text { El estudiante en sus prácticas } \\
\text { toma en cuenta los puntos de } \\
\text { vista de los demás de forma } \\
\text { constructiva contextualizando } \\
\text { lo aprendido usando ejemplos } \\
\text { prácticos. }\end{array}$ & 0 & 2 & 13 \\
\hline & & & $\begin{array}{l}\text { El estudiante solicita } \\
\text { opiniones críticas sobre su } \\
\text { trabajo para mejorar su grado } \\
\text { de rendimiento }\end{array}$ & 0 & 2 & 13 \\
\hline
\end{tabular}


Fuente: elaboración propia

\section{Interpretación de resultados Tabla 1: Observación a los estudiantes}

La mayoría de la población, son competentes para resolver problemas y lograr los objetivos propuestos; tan solo un estudiante no utiliza las competencias en un contexto determinado para la consecución de los objetivos propuestos. En cuanto, al estudiante que no ha desarrollado las competencias para resolver problemas es necesario de parte del docente diseñar la estrategia pertinente que le facilite al educando desarrollar esta competencia, ya que es función de la universidad como del docente "el desarrollo y mejoramiento de las competencias en los estudiantes de todas las carreras" (Ochoa, 2012).

En la categoría dos, aplicación de los conocimientos, en los dos contenidos de análisis, las calificaciones de los estudiantes fueron idénticas, 1 estudiante fue calificado en Insatisfactorio, por lo tanto, no utiliza el proceso apropiado para solucionar situaciones complejas. En cambio, 4 estudiantes, su calificación fue Bueno y 10 en Ejemplar. Dicho de otra manera, 14 estudiantes aplican los conocimientos previos y adquiridos para la consecución de los objetivos propuestos y hace uso de eso conocimientos a la hora de tomar de decisiones complejas; únicamente 1 estudiante no aplica los conocimientos ni hace uso de estos para conseguir los objetivos propuesto ni para tomar de decisiones complejas.

En la tercera categoría, Toma de decisiones, las calificaciones en los dos contenidos de análisis fueron similares, 1 de los participantes se calificó en Insatisfactorio; 2 en Bueno y 12, fueron calificados en Ejemplar. Esto significa que el estudiante es capaz de tener iniciativa, tomar decisiones sencillas, coherentes y sabe justificarlas con criterio en situaciones complejas. Solo 1 participante, no utiliza esta competencia en un contexto determinado y no saber tomar decisiones. El estudiante evidencia una falla en su proceso y es preciso corregirla ya que deben estar en "capacidad de constituirse en aprendices por el resto de sus vidas, comprometidos y en capacidad de desarrollar un aprendizaje y una reflexión permanentes con el propósito de desarrollar su comprensión del mundo y su papel en el mismo" (M.E.N, 2016, p.21).

En la cuarta categoría de la guía de observación, denominada Aprendizaje, en los dos contenidos de análisis, los resultados fueron de 12 participantes calificados en Ejemplar y 3 calificados en Bueno; de modo que, en esta categoría los estudiantes demostraron tener habilidad y destreza para solicitar opiniones, tenerlas en cuenta y mejorar su grado de rendimiento contextualizando lo aprendido y usando ejemplos prácticos.

\section{Tabla 2}

\section{Resultados obtenidos de la entrevista semiestructurada a los docentes}

\begin{tabular}{|c|c|l|}
\hline Categoría & Preguntas & \multicolumn{1}{c|}{ Respuestas } \\
\hline \multirow{4}{*}{$\begin{array}{c}\text { Concepciones } \\
\text { teóricas }\end{array}$} & $\begin{array}{c}\text { ¿Qué es una } \\
\text { competencia? }\end{array}$ & $\begin{array}{l}\text { Docente 1: "Son las destrezas que desarrolla una persona } \\
\text { con las cuales actúan en el mundo" }\end{array}$ \\
\cline { 3 - 3 } & $\begin{array}{l}\text { Docente 2: "Son las capacidades y conocimientos que los } \\
\text { seres humanos estructuran para pensar y actuar". }\end{array}$ \\
\cline { 3 - 3 } & $\begin{array}{l}\text { Docente 3: "Es la sincronización entre los conocimientos } \\
\text { las habilidades y la actitud con que cuenta una persona para }\end{array}$ \\
\hline
\end{tabular}




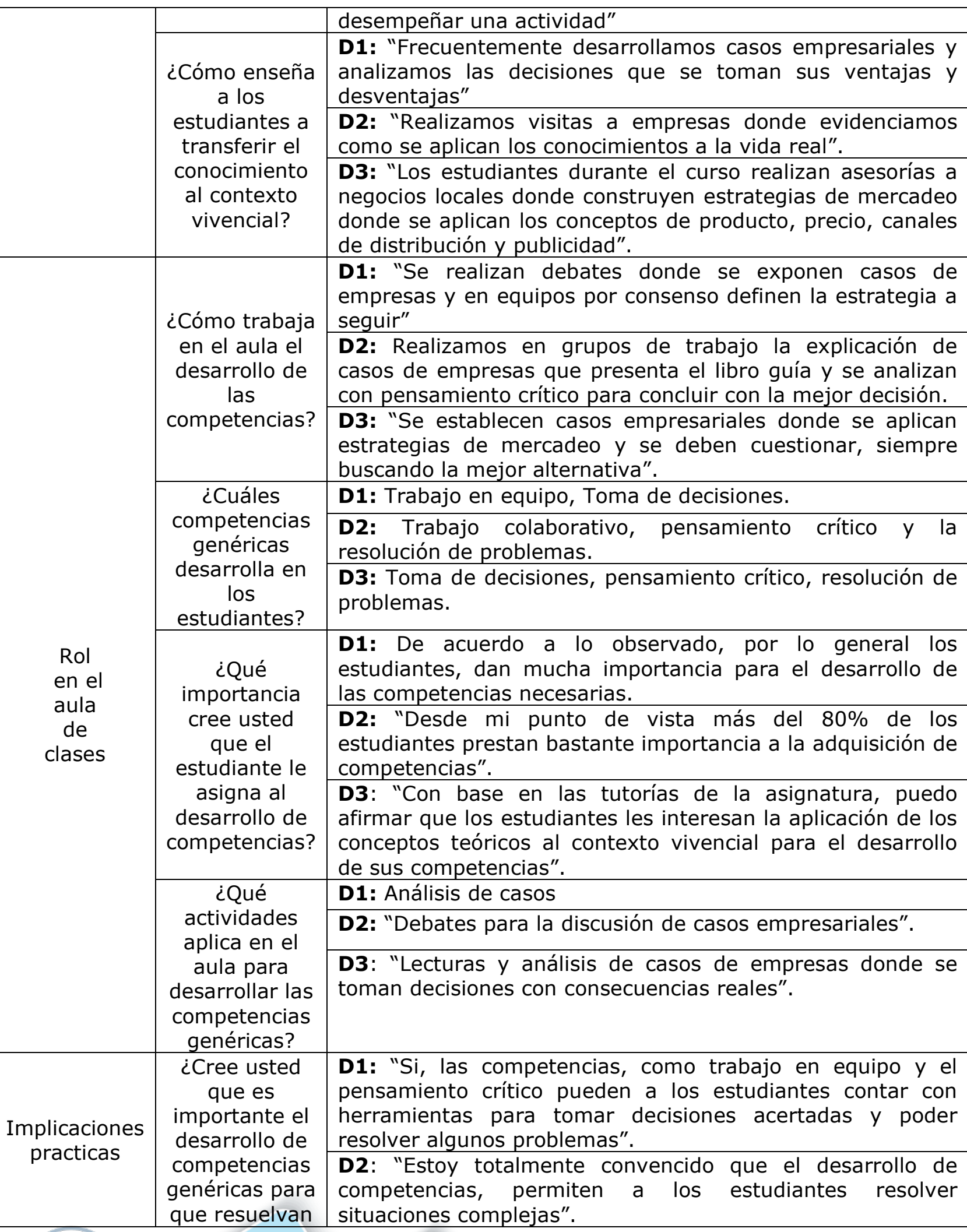




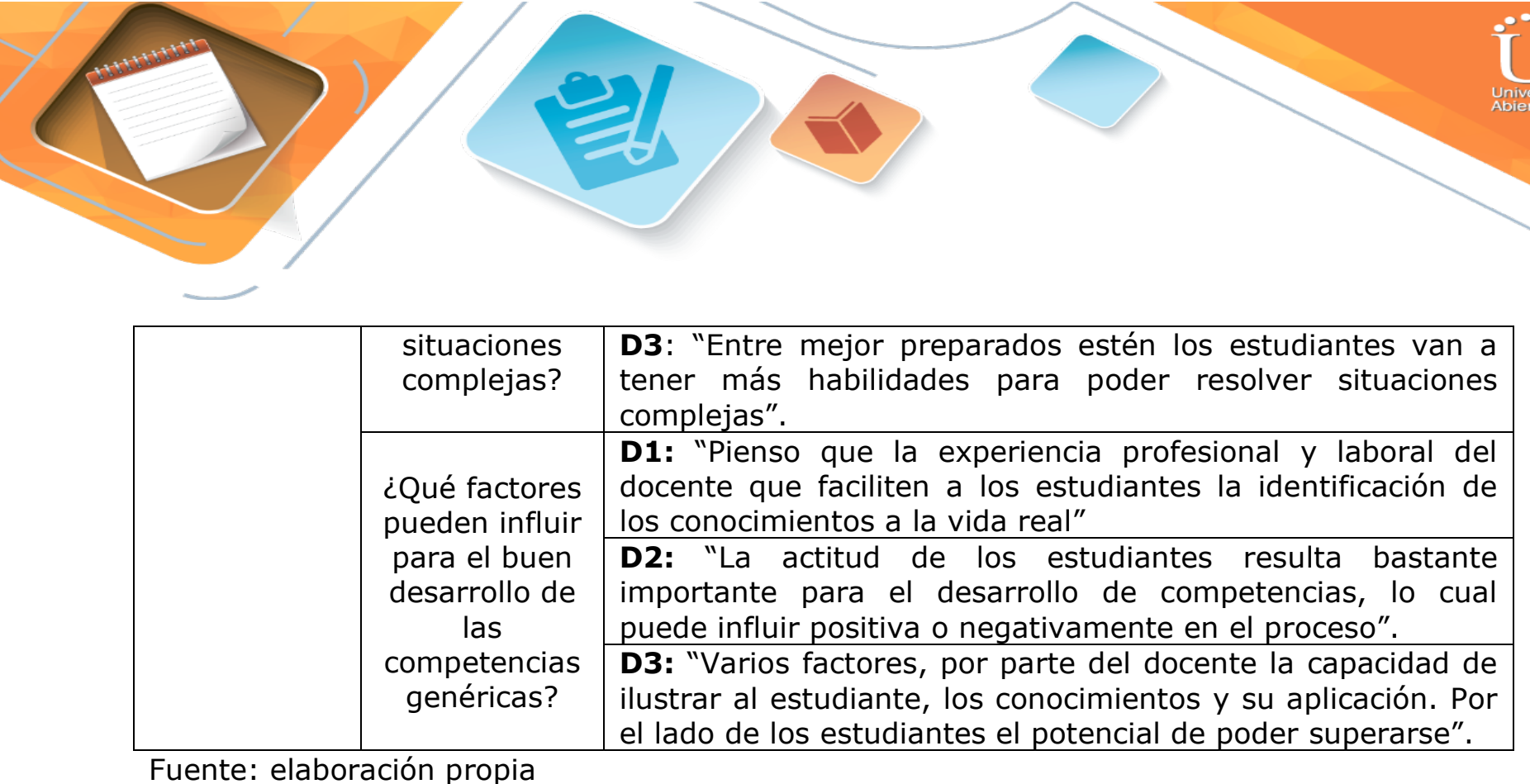

Fuente: elaboración propia

La entrevista se dividió en tres categorías: la primera categoría, concepciones teóricas tiene dos preguntas; la segunda categoría, rol del docente en el aula de clases, con cuatro preguntas y la tercera categoría, implicaciones prácticas con dos preguntas. En la tabla están las respuestas textuales de los tres docentes.

En las respuestas a las dos primeras preguntas de la categoría concepciones teórica, es claro que los docentes manejan el concepto de competencia y los enseñan con seguridad y dominio a sus estudiantes. Además, los tres docentes emplean casos pertinentes para el desarrollo de estas competencias y la forma como los estudiantes debe transferirlas a su contexto vivencial. En el aula han estado trabajando, la toma de decisiones, el trabajo en equipo y resolver problemas, un trabajo acertado ya que "una competencia es un saber hacer complejo, que se manifiesta en la actuación efectiva sobre una situación problemática, para cuya solución se movilizan integradamente diferentes habilidades, conocimientos y actitudes" (Daza y Charris, 2015, p.3).

Vale la pena mencionar que dentro de los resultados evidenciados con las respuestas de la entrevista se resalta, la aplicación de diferentes estrategias pedagógicas como los debates, análisis de casos empresariales y prácticas empresariales, que fortalecen el desarrollo de las competencias genéricas como, aplicar los conocimientos en la práctica, la capacidad para identificar, planear y resolver problemas y la toma de decisiones y de igual manera facilita la transferencia de los conocimientos del aula al contexto vivencial.

\section{CONCLUSIONES}

La integración y desarrollo de las competencias genéricas y específicas en el aula resultan pertinentes para mejorar el proceso de formación, adaptación y desempeño de los educandos en el contexto laboral y vivencial. Igualmente, se confirmó lo valioso que resulta el desarrollo de las competencias y los aportes que éstas hacen en el desempeño de los estudiantes. Por ejemplo, con la guía de observación se halló, que, si a los estudiantes se les enseña a trabajar por competencias, ellos logran utilizar el proceso apropiado para solucionar situaciones nuevas y complejas. Esto significa que los estudiantes aprenden a ser capaces de tener iniciativa, tomar decisiones sencillas, coherentes y saber justificarlas con criterio en situaciones complejas. 
Para los estudiantes, las competencias genéricas al ser un conocimiento y una habilidad adquirida o enseñada les ayudan a transferir los conocimientos a su contexto vivencial porque se convierten en una herramienta práctica para resolver problemas, tomar decisiones y trabajar en equipo en cualquier contexto de su vida cotidiana. Trabajar actividades en el aula tales como el análisis de casos empresariales y debates, despiertan el interés de los estudiantes por desarrollar esas habilidades y capacidades para enfrentar situaciones adversas y tomar la mejor decisión que resuelva el problema que se le presente. Estas actividades los concientiza de la importancia de estas competencias, y esto ha hecho que la actitud de los educandos cambie, siendo más receptivos, lo cual es un factor clave porque inciden directamente en la consecución de las metas y objetivos trazados.

La universidad y demás instituciones deben ejecutar proyectos transversales para que estas competencias genéricas se trabajen en todas las disciplinas y materias. De igual forma, el docente debe abandonar toda practica de enseñanza tradicional, el estudiante ya no requiere memorizar información exacta encontrada en libros u otros medios, lo que necesita es que le enseñen cómo aplicarla en su vida diaria y laboral.

A nivel nacional el Ministerio de Educación Nacional de Colombia (2016) le ha apostado a la aplicación de unas competencias genéricas que sean transversales a todos los niveles educativos para la formación de estudiantes competentes y productivos, que estén acordes con los constantes cambios de este mundo globalizado. Por parte de esta investigación se dejó en evidencia lo importante que es desarrollar en los estudiantes competencias genéricas tales como: aplicar los conocimientos en la práctica, resolver problemas y tomar decisiones. A partir de lo observado en los estudiantes en el trabajo de campo, estas competencias son benéficas para su contexto laboral y vivencial.

Por consiguiente, la presente investigación confirma lo imperioso de trabajar las competencias en el aula; no obstante, se debe ser cuidadoso y tener en cuenta lo manifestado por Rodríguez Izquierdo (2013), de no convertir la enseñanza universitaria en un proceso meramente mercantilista.

Es preciso recordar que las competencias, es un saber en acción en una situación determinada, y que, por ser un saber, unas habilidades, es posible transferirla a la realidad inmediata sin ningún inconveniente. Lo más importante, para que el estudiante realice esta transferencia de sus conocimientos a un contexto real, es contar con la asertiva orientación del docente en el aula, que mantenga motivado al estudiante a desarrollar sus competencias para que ellos no olviden lo valiosas que son en cualquier situación, ya sea laboral, académica o familiar. Todo lo anterior, se vincula al entorno laboral, donde las competencias que se forman desde el aula, se fortalecer hasta la formación profesional, por lo que las competencias profesionales (Tovar Trujillo, Vargas Paredes, \& González González, 2018), fortalecen sus habilidades, y especializarse en su área, para aportar a la eficacia y efectividad de las empresas, de las organizaciones, con el propósito de posicionarse como los mejores de su negocio. Es así como, las competencias son esenciales para el futuro de los estudiantes y su proyecto de vida en el desarrollo de sus actuaciones desde los diferentes contextos.

Las estrategias pedagógicas de los docentes permiten desarrollar competencias genéricas en el aula, le facilita al educando, ya sea de forma individual o en equipo trabajar el pensamiento crítico a partir del análisis de un problema o de una tomada de decisión. De igual forma, al abordar casos empresariales el estudiante pone en práctica estrategias donde 


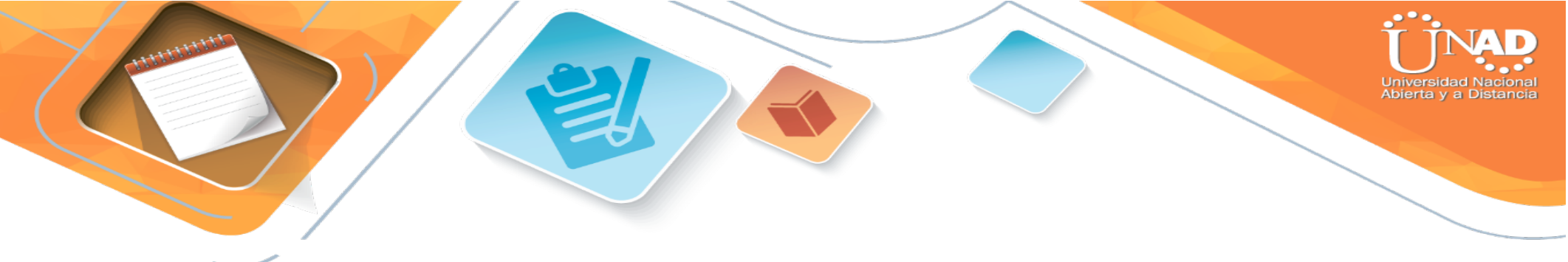

Tovar Trujillo, S., Vargas Paredes, S., \& González González, G. (2018). Toma de decisiones en la gestión del sector agroindustrial del bizcocho de achira en Huila (Colombia). Espacios, 4- 15 